\title{
Acute Disseminated Encephalomyelitis Secondary to Herpes Virus Infection in a Child
}

\author{
Z Ouassou' ${ }^{1}$, Y Motiaa' ${ }^{2}$, A. Bentalha ${ }^{3}$, A. El Koraichi ${ }^{4}$, S. E. El Kettani ${ }^{5}$
}

\begin{abstract}
The acute disseminated encephalomyelitis ADEM is a rare form of encephalitis. We report a case after herpes infection. The diagnosis was based on the existence of post-infectious and neurological signs and demyelinating reaching the white matter in the magnetic resonance imaging MRI. The outcome was favourable after treatment with high-dose steroids.
\end{abstract}

Key words: Encephalomyelitis, Infection, Herpes, Demyelinating, White matter.

\section{Introduction}

$\mathrm{T}$ he acute disseminated encephalomyelitis (ADEM) is a diffuse autoimmune demyelination of the central nervous system, secondary to a viral infection or vaccination. Many infectious agents have been reported. An infectious history, in the two weeks preceding the neurologic event, is observed in nearly $60 \%$ of child cases. Typically, the clinical picture includes signs of meningoencephalitis with white matter abnormalities, visible via MRI. The treatment is based on a high-dose corticosteroid therapy. A case of ADEM was observed in a two year boy, associated with a herpes virus infection.

\section{The Case}

A two-year old boy was referred to the service of infectious diseases following a febrile wakefulness that occurred abruptly. The patient's notable medical antecedent was an HSV1 encephalitis, treated before 4 weeks with a good evolution. There was no report of a recent vaccination or intoxication.

At the time of admission, the child showed a somnolence with a Glasgow score at $12 / 15$ and a fever of $40^{\circ} \mathrm{C}$. Capillary blood glucose was at $0.7 \mathrm{~g} / \mathrm{l}$. The neurological examination revealed a meningeal stiffness, a bilateral pyramidal syndrome and sphincter disorders. The pupils were reactive and symmetrical and there were no signs of cranial nerves injury. Brain CT scan revealed a diffuse parenchymal hyper density. The complete blood count (CBC), the C-reactive protein (CRP), the lumbar puncture (LP), the blood electrolytes and liver function were all normal.

On the $3^{\text {rd }}$ day, a neurological deterioration (with swallowing disorders) called for the intubation of the patient and an urgent brain CT scan; diffuse hyper dense areas were observed without
${ }^{1}$ Dr. Zakaria Ouassou, School of Medicine, Mohamed V University, Rabat, Morocco, ${ }^{2}$ Dr. Youssef Motiaa, School of Medicine, Mohamed V University, Rabat, Morocco, ${ }^{3}$ Dr. Aziza Bentalha, Pediatric ICU, Children Hospital, Rabat, Morocco, ${ }^{4}$ Dr. Alae El Koraichi, Paediatric ICU, Children Hospital, Rabat, Morocco, ${ }^{5}$ Dr. Selma Ech-Chrif El Kettani, Paediatric ICU, Children Hospital, Rabat, Morocco.

\section{Address for correspondence \\ Zakaria ouassou \\ E-mail: zakaria.ouassou@gmail.com}

\section{How to cite}

Z Ouassou, Y Motiaa, A. Bentalha, A. El Koraichi, S. E. El Kettani. Acute Disseminated Encephalomyelitis Secondary to Herpes Virus Infection in a Child. J Nepal Paediatr Soc 2017;37(2):191-193.

doi: http://dx.doi.org/10.3126/jnps.v37i2.16976

This work is licensed under a Creative Commons Attribution 3.0 License. 
meningeal contrast. A second lumbar puncture was made the same day and found a clear cerebrospinal fluid lymphocytosis (CSF). The CSF culture was negative. Brain MRI evidenced diffuse areas in white matter hyper intensities of the brain, suggesting a demyelination of the white matter. The serologic profile of HSV1 realized was in favor of a former immunity. The mycoplasmal pneumonia serology was negative. The diagnosis of an ADEM secondary to HSV1 infection was retained. A methylprednisolone bolus therapy was initiated immediately at the dose of $10 \mathrm{mg} / \mathrm{kg} / \mathrm{d}$. Initially, the evolution was stationary (Glasgow at 6/15, convulsive status). Despite the absence of any clinical progress in the beginning and the presence of sequels at imaging, the symptomatic treatment eventually allowed the recovery of an almost complete autonomy with ambulation, communication and recovery of the higher functions.

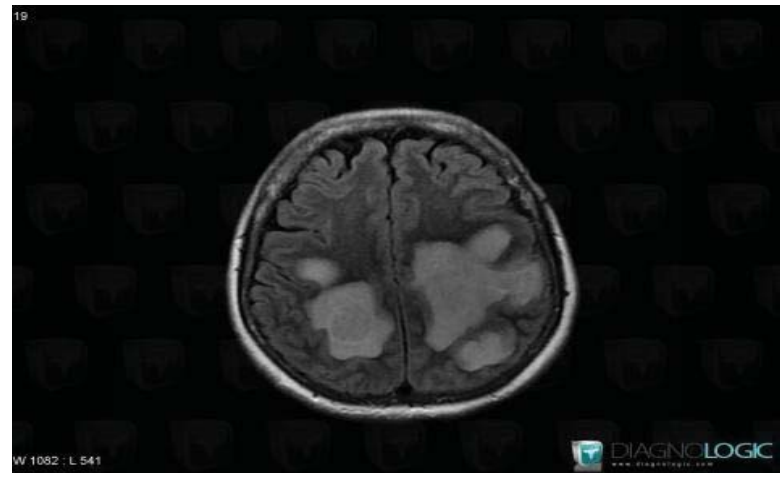

Fig 1: Brain MRI Axial $\mathrm{T}_{2}$-weighted fluid-attenuated inversion recovery images show large bilateral and asymmetric hyperintensity, in the semioval centers and the sub-cortical white matter predominantly in the parieto-occipital areas

\section{Discussion}

ADEM is defined as a multifocal demyelinating inflammatory attack mainly involving the white matter of the central nervous system (CNS). It is mediated by an autoimmune mechanism and occurs generally after an infection or vaccination. The causal agent in our case was an infection by HSV1. One of the mechanisms explaining the ADEM lesions relates to the homology of structure between the triggering factor and the host's myelin antigens. We refer to it as molecular mimicry. Another hypothesis involves the infection of the CNS by a neurotropic pathogen, causing brain damage, a rupture of BBB and the passage in the blood of CNS antigens $\mathbf{s}^{1,2}$

The clinical features are abrupt or rapidly progressive and involves encephalopathy and hemispheric or spinal focal neurological signs. An encephalitic picture, associating consciousness disorders, convulsions, fever, and meningeal stiffness, is common. Consciousness disorders are found in 19 to $69 \%$ of patients ${ }^{3,4}$. The most severe cases can lead to a state of coma with signs of decerebration. Meningeal stiffness is reported in 5 to $44 \%$ of the cases depending on the series. Fever is most frequently found (43-52\%). Convulsions are reported in 13 to $35 \%$ of the cases, as noted in the case in our patient.

Focal deficit signs are frequent. Hemiplegia is noted in approximately $75 \%$ of cases. We can find a uni or bilateral pyramidal syndrome in 60 to $95 \%$ of the cases and lesions of the cranial nerves in 23 to $50 \%$ of cases ${ }^{5}$. The particularly evocative visual lesion is characterized by an optic neuropathy. The spinal cord and peripheral nervous system lesions are likely but rare in children ${ }^{6}$. Our case showed spinal cord lesion.

The analysis of CSF enables to rule out an infection of the central nervous system and can reveal nonspecific abnormalities. The intrathecal synthesis of $\operatorname{lgG}$ is found in 3 to $29 \%$ of cases ${ }^{7}$. There is no biological inflammatory syndrome, but discrete abnormalities can be found in the blood of the neutrophil polynucleosisor lymphopenia type. The blood test of our patient did not show any abnormalities except for a persistent CSF high protein. The brain CT scan is a key element in the diagnosis, as the lesions are typically multiple and scattered, and affect predominantly the white matter of the brain. Diffuse lesions can be found in $30 \%$ of cases ${ }^{7}$.

The therapeutic approach is based on immunomodulatory treatments. The most frequently used treatments include intravenous corticosteroids (CT), versatile immunoglobulins (IVIG) and plasma exchange (PE). The corticotherapy boluses allowed for a significant improvement in our case. Plasma exchanges were reported in few cases, the place of this treatment remains to be defined, but it is seemingly justified in the case of non-responsiveness to corticotherapy, especially with patients hospitalized in intensive care.

Presently, ADEM induced mortality does not exceed $5 \%$. The presence of convulsions or deep consciousness disorders at admission could be associated with a negative prognosis. The most frequent neurological sequelae are focal deficits, ataxia, or visual problems. Cognitive and behavioural disorders are identified in 6 to $50 \%$ of the patients in the paediatric series ${ }^{7}$. After one year, our patient did not show any behavioural disorders, but remained with a sequelae epilepsy and focal deficits. 


\section{Conclusion}

ADEM diagnosis needs to be systematically put forward in presence of an unexplained acute encephalitis, especially if a notion of recent infection is found in the background. The treatment of ADEM is based on a high-dose corticoidotherapy, possibly associated with polyvalent immunoglobulins or plasma exchange. The prognosis is generally favourable under treatment, and recurrences can nevertheless arise with time.

\section{References}

1. Dale RC, de Sousa C, Chong WK, Cox TC, Harding B, Neville BG. Acute disseminated encephalomyelitis, multiphasic disseminated encephalomyelitis and multiple sclerosis in children. Brain 2000;123(Pt 12):2407-22. DOI: https://doi.org/10.1093/ brain/123.12.2407

2. Tenembaum S, Chitnis T, Ness J, Hahn JS. Acute disseminated encephalomyelitis. Neurology 2007;68:S23-36. DOI: https://doi.org/10.1212/01. wnl.0000259404.51352.7f

3. Dale RC. Acute disseminated encephalomyelitis. Semin Pediatr Infect Dis 2003;14:90-95.
4. Menge $\mathrm{T}$, Hemmer $\mathrm{B}$, Nessler $\mathrm{S}$, Wiendl $\mathrm{H}$, Neuhaus O, Hartung HP, et al. Acute disseminated encephalomyelitis: an update. Arch Neurol 2005;62:1673-680. DOI: https://doi.org/10.1007/ s11908-008-0050-7

5. Garg RK. Acute disseminated encephalomyelitis. Postgrad Med J 2003;79:11-17. DOI: http://dx.doi. org/10.1136/pmj.79.927.11

6. Love S. Demyelinating diseases. J Clin Pathol 2006;59:1151-59.DOI:10.1136/jcp.2005.031195

7. Krupp LB, Banwell B, Tenembaum S. Consensus definitions proposed for pediatric multiple sclerosis and related disorders. Neurology 2007;68:S7-12. DOI: https://doi.org/10.1212/01.wnl.0000259422.44235.a8 